\title{
Modelos lingüístico-literarios vascos anteriores al 68
}

\author{
PATRICIO URQUizU \\ UNED \\ patriurkizu@euskalnet.net \\ Recibido: mayo de 2013. Aceptado: junio de 2013
}

\begin{abstract}
Resumen: Tras unas consideraciones críticas sobre el tópico de que el sistema literario vasco es una isla, ya que la literatura siguiendo a Lotman es un sistema supralingüístico, se presentan los cuatro modelos lingüístico-literarios anteriores al Congreso de la Unificación de la Lengua Vasca de Aránzazu en 1968: el de Leizarraga, el de Axular, el gipuzkera osatua [guipuzcoano completado] y el de otros dialectos. Así mismo se presentan algunos aspectos particulares de los modelos según los géneros: poesía, narrativa, ensayo y teatro.
\end{abstract}

Palabras clave: Sistemas literarios. Modelos lingüístico-literarios en euskera.

Laburpena: Gogoeta egin ondoren aurreiritzi zenbaitez, zeinetan kontsideratzen baita euskal literatura uharte bat bailitzan, kontuan hartu gabe literatura egitura supralinguistikoa dela, Lotman-ek hain justuki dioen bezala, 1968an Arantzazuko Batasunaren Biltzarrean aurkeztu aitzin eman diren eredu linguistiko-literarioak aztertzen dira: Leizarragarena, Axularrena, gipuzkera osatuarena eta zenbait euskalkienak. Halaber eredu hauen moldekatze berezia poesia, narrazioa, saioa eta teatroa generoetan ikertzen da.

Gako hitzak: Literatura egiturak. Eredu linguistiko-literarioak euskaraz.

\author{
Recorda sempre això, Sepharad, \\ Fes que siguin segurs els ponts del dialèg, \\ I mira de comprendre i estimar \\ Les raons i les parles diverses dels teus fills
}

La pell de Brau (Salvador Espriu)

Harkaitz Cano, joven escritor y licenciado en Derecho, — caso que demuestra cómo se puede vivir sólo de la literatura en euskera sin ejercer la abogacía-, que participó en una de las últimas Ferias de Frankfurt en el acto organizado por la Asociación de Editores en Lengua Vasca, citando al poeta inglés Auden, y sus Cartas desde Islandia, hablaba de «la soledad de la literatura vasca» y la comparaba con la soledad de las islas. 
Es una imagen y una idea similar la que desarrolla María José Olaziregi [2001:325-336] en su artículo sobre la narrativa vasca contemporánea. «El sistema literario vasco es una isla», y la novela vasca contemporánea tiene un carácter «insular». Y al buscar las razones del hecho, piensa que además de las razones extraliterarias (falta de curiosidad de la institución literaria española hacia las literaturas periféricas, - $-\mathrm{y}$ no digamos de la institución literaria francesa-, o el continuo bombardeo de noticias que asocian lo vasco únicamente al terrorismo) tendríamos que encontrar en el propio sistema literario vasco las razones de este aislamiento.

Una isla a la que arriban cada día más barcos pero de la que zarpan pocos. Es decir, que se traduce mucho al euskera pero poco del euskera a otros idiomas. Las razones son muy complejas. En primer lugar habría que tener cuenta de las dificultades inherentes a toda traducción. Ya que como sabéis toda traducción exige: a) un dominio perfecto de ambas lenguas, es decir, de la de partida y de la de llegada, b) un conocimiento profundo de la materia de que se trata, y c) ser un buen escritor y acertar con el tono.

Podríamos hablar del caso chino y ver cómo lo han resuelto. Uno de los mayores y mejores traductores al chino era un gran poeta que ni conocía inglés ni francés. Un conocedor de estos idiomas le vertía al chino las obras de Dickens, Balzac o Dumas, y él les daba el hálito poético. Por eso cuando fue Mitterand a China el presidente chino le habló de un autor de segunda fila que en China estaba considerado como de los mejores de Occidente, lo que extrañó en gran medida al presidente francés. Se trataba de un milagro o simplemente del arte chino de la traducción, según Simon Leys (1998).

Hay quien considera que para qué traducir del euskera. Claro que las razones extraliterarias tienen en esta postura mucha culpa, pero también hay autores que piensan que si su obra se traduce disminuirá el número de lectores en euskera, por lo que la lengua ya en dificultades correrá aún más riesgos.

Opino de todos modos, que existe también un problema de solidaridad cultural, y que de ese modo tal vez se pudiera pagar algo la deuda adquirida con los otros, con los demás escitores y con las otras culturas, y que el conocimiento del País Vasco no fuera de ciencia-ficción, como se podría deducir si se leyeran exclusivamente los periódicos madrileños, sino más profundo, con esas matizaciones y detalles que sólo da la literatura.

Los términos habituales con los que se adjetivan la lengua vasca y su literatura son, insular, periférica y minoritaria, pero éstos epítetos creo que merecen ser puntualizados y revisados, pues no se corresponden en mi opinión exactamente con la realidad y creo que dependen fundamentalmente de dónde se ubique la mirada.

Se ha repetido con frecuencia que la lengua vasca es una lengua isla, y hasta llegó esta idea a Saussure que consideraba que on ne pouvait rien tirer du basque parce que étant isolé, il ne se prête à aucun comparaison. Es decir, que nada se podía deducir del euskera porque siendo una lengua aislada no se prestaba a ninguna comparación. 
Contra esta idea ya se alzó Michelena en su obra Lenguas y protolenguas de 1963, —algo así como El Discurso del Método de la Reconstrucción Lingüística-, al considerar que el euskera no es una lengua isla, y que sí se puede comparar con el latín y las lenguas romances vecinas, y sacar conclusiones importantes para su Historia.

El asentamiento de distintas lenguas y culturas durante la Edad Media en el territorio vascón, y el ser el País Vasco un lugar de paso hacia Santiago de Compostela, uno de los lugares de religiosidad y peregrinación más importantes de la Edad Media lógicamente ha hecho que quien más quien menos, haya dejado su huella en los territorios por los que pasaban. Un estudio a fondo tanto del léxico como de la toponimia y antroponimia nos llevaría a concluir sobre la importancia del multilingüismo en estos lares, y descartar la idea tópica de su carácter insular.

En este sentido, pero refiriéndonos a la literatura, hay que recordar aquello que dice René Wellek. Que es evidentemente falsa la idea de «una literatura nacional aislada», porque la literatura occidental, al menos, forma una unidad, una totalidad, ya que no sólo emigran temas y motivos, formas y géneros, ideas y símbolos sino que hay un desarrollo general europeo y americano de la literatura.

Por lo que Yuri Lotman ve la literatura (1978) como una totalidad, es decir un sistema «supralingüístico» en términos de D. Fokkema (1998: 153).

Con respecto al término periférico, me parece que corresponde al mundo de la geografía, de la circulación o de los ordenadores, y no realmente al de las culturas, porque como ya lo expresó el ensayista valenciano Joan Fuster (1969), las lenguas del estado español y sus culturas, excepto la castellana, han sido postergadas, y sus hablantes «marginados, convertidos en episodio apendicular aberrante, o, a lo sumo, complementario».

En la misma recopilación de estudios citada arriba aparece un trabajo de Steven Tötösy, en el que al hablar de centro y margen, piensa que aunque es obvio que el centro ejerce poder e influencia ello debe ser conciliable con la comprensión de las literaturas de los márgenes.

No sólo conciliable sino muy saludable (Estos críticos modernos americanos están descubriendo la tortilla de patata, y pronto nos la van a vender como el último hallazgo...).

Así pues, hay que tener en cuenta lo que respondió Txillida cierta vez a un periodista de El País, cuando le preguntó si era nacionalista, «yo veo el mundo desde San Sebastián». Como Baroja que lo veía desde Donostia, muy diferente al mundo que veía Unamuno, desde Salamanca por supuesto. Todo depende del punto de vista que se tome. Como dice uno de los personajes en el comienzo de una novela, de cuyo nombre de autor no quiero acordarme, «no perdamos la perspectiva».

Y la perspectiva es, que Uno, cada uno es el centro de sí mismo y el Otro es la periferia. Y si hablamos de centros culturales europeos y del mundo, y si consideramos hoy como tales a Berlín, París, Londres o Nueva York, se halla 
Madrid tan a su periferia o más que Donostia, o Barcelona, o Valencia. Claro que también hay que tener en cuenta que un porcentaje altísimo de la cultura que se recibe aquí referente a estos focos es en castellano, pero como decía Michelena el castellano también, o el francés (añado) es patrimonio de los vascos.

Además, es evidente que hoy día incluso estando en la aldea más remota puede uno participar de la cultura mundial, sin tener que soportar a los tertulianos madrileños.

Minoritario es otro de los clichés que están en boga, y que a veces suele ser el camino para la exclusión y minusvaloración. He de confesar que a mí no me importa en absoluto ser autor de una lengua minoritaria y escribir for the happy few, para la felicidad de unos pocos. En concreto cuando me propuse escribir en euskera a los diecisiete años tras ganar un premio en castellano ya sabía cuál era mi destino, y cuando escribí una novela-crónica de la guerra civil, sólo pensé que podía dicho trabajo hacer pensar algo a los de mi pueblo. El día de la presentación no cabía la gente en el salón consistorial y hubo quien leyó por primera vez en su vida un libro en euskera y le gustó y además al parecer casi no tuvo ningún problema de comprensión al leerlo. Además uno al cabo de los años, a pesar de que haya publicado muchos libros, y tal vez por eso mismo, se da cuenta que es un escritor corriente, normal y no ningún genio. Y si es evidente que el euskera no entra en el ranking mundial de lenguas habladas entre las diez primeras, sí entra entre las cincuenta y uno, y si nos referimos al uso de la lengua en Internet nos hallamos entre las treinta y tantas más utilizadas.

Es cierto que las tiradas de algunos libros han bajado, pero esto también es algo general. De un libro de cuentos que publiqué el año 84 se vendieron más de 3.000 ejemplares, lo que quiere decir que en cifras relativas haciendo el cambio al número de castellano-hablantes equivale a más de un millón. Claro que también de otro libro que publiqué hace unos años (2000), un epistolario de exilio sólo se publicaron 300 ejemplares.

Injusticias literarias.

Por lo tanto el tópico de que el euskara sea una lengua isla, y pertenezca a una literatura periférica y minoritaria es bastante cuestionable, o por lo menos puntualizable.

Una lengua, la más antigua del continente europeo, con 750.000 hablantes aproximadamente, que los más optimistas alargan hasta el millón y los más pesimistas bajan al medio, según los grados de conocimiento y uso. 2.000 libros producidos el año 2007 contra 732 de 1979, 70 de 1969 y 10 en el 1949. Como se ve una irresistible ascensión. Y de la que la parte del león se la lleva la narrativa con un $60 \%$ de la producción. Cinco novelas en los 40, ocho en los 50, diecisiete en los 60, y...doscientas en el 2000.

Pero hablemos de los modelos lingüístico-literarios anteriores al 68, que es el tema impuesto por mi amiga la profesora Maite Etxenike.

Durante los días 3, 4 y 5 de octubre de 1968 se celebró en el Monasterio franciscano de Aránzazu un Congreso de la Academia de la Lengua Vasca-Eus- 
kaltzaindia, en el que participé siendo muy joven, ya que en aquella época era estudiante de Filología Románica en Salamanca. Se trataba de aprobar un informe escrito por el profesor de Lingüística Preindoeuropoea de la Universidad de Salamanca y miembro de Euskaltzaindia el Dr. Luis Michelena, sobre la Unificación del euskera escrito. Se titulaba Euskeraren Batasuna (Donostia, 1969), y se trataba de poner las bases de unificación en principio de la ortografía vasca. Seguía las pautas ya señaladas en el Congreso de Bayona de 1964 y que algunos de los escritores más importantes del momento seguían y proponían.

Tenía varios puntos importantes:

1. Bases.

2. La Ortografía.

3. Sobre las palabras antiguas.

4. Sobre los Neologismos.

5. Morfología.

6. Sintaxis.

Era evidente que es cuestión de vida o muerte la unificación de una lengua, y que no se podían seguir utilizando para la palabra cama siete formas diferentes, sino que entre todas las variantes había que elegir aquella de más raigambre literaria, cual podía ser la forma ohe. Así mismo entre las formas dut / det / dot entre otras para decir tengo había que elegir una, que tuviera más aceptación. Fue un congreso muy disputado sobre todo, porque se centró a veces en vez de en los principios programáticos de base, en el uso o no uso del grafema $<\mathrm{h}>$.

De todos modos la consideración de Mitxelena de que la unificación para la enseñanza del euskera escrito, no del hablado ni del literario había de basarse sobre dos pilares, uno de tipo literario y otro de tipo sociolingüístico fue bastante bien recibida.

En primer lugar se hallaba el modelo laburdino, lapurtar klasikoa, y la escuela de Donibane Lohizune [Saint-Jean de Luz ]- Ziburu-Sara, representado en concreto por Axular, el gran escritor del siglo XVII, que mostraba las pautas a seguir. Esta propuesta venía avalada por diversos artículos de Luis Villasante.Y más tarde una vez hecha la unificación de la ortografía había que elegir entre los diversos modelos de la morfología verbal, aquel que tuviera más aceptación social, por lo que se optó por la morfología del verbo guipuzcoano con algunos aderezos labortanos.

Era como señalaba Pierre Lafitte, —que había editado en 1944 y reeditado en 1966 una Grammaire basque (Navarro-labourdin littéraire), donde en la balanza de los dialectos, la norma en contra de la tradición literaria labortana se inclinaba hacia el bajo navarro de mayor peso sociolingüístico-, con una imagen culinaria, cuestión de hacer una buena mayonesa, para lo que había que saber evidentemente dosificar bien el aceite y los demás ingredientes. 
¿Pero cuáles eran los modelos lingüísticos y literarios anteriores y cuál su prestigio? Había en principio cuatro modelos lingüísticos en debate:

a) El modelo de Leizarraga.

b) El modelo de Axular

c) El modelo del gipuzkera osatua [el guipuzcoano completado].

d) Otros modelos dialectales.

Y a nivel literario según los diversos géneros los modelos serán diferentes:

a) Poesía popular / poesía culta.

b) El cuento tradicional / el cuento literario / la novela.

c) El ensayo religioso / didáctico-filosófico-político.

d) El teatro popular / el teatro culto.

Joanes Leizarraga traductor del Nuevo Testamento (1571) y de opúsculos protestantes (1559...) según J.M. Olaizola nació en 1506 y murió en Labastida en 1601. En el prólogo dedicado a los vascos, señala que como todo el mundo sabe en Heuscal-Herria casi de una casa a otra hay diferencia y diversidad en las maneras de habla, y que por ello ha intentado ahalic guehienic guciey eraciteari iarrequi içan gaitzaitza, eta ez choil edocein leku iaquineco lengoage bereciri [expresarse de modo que el mayor número pudiera entenderle, y no en la manera de lenguaje de un lugar concreto]. Y así para ayudar a los suletinos en la comprensión del texto escribe al final del Nuevo Testamento un breve léxico Çuberoaco herrian usançatan eztiraden hitz bakoitz batzu hango ançora itzuliac [Algunas palabras que no se usan en Zuberoa traducidas al modo de allí].

Fue realmente una pena que el modelo lingüístico propuesto por Leizarraga y su grupo de traductores no tuviera el éxito que se merecieron por la corrección de presupuestos en los que se basaban, como lo tuvo Lutero en Alemania y que sirvió para la unificación del alemán, pero como es sabido en las catolicísimas Francia y España, los protestantes, calvinistas y hugonotes fueron brutalmente perseguidos y sus obras pasto de la hoguera.

Luis Villasante el año 1952 publicó un largo artículo de 70 páginas titulado Literatur-euskera, laphurtarr klassikoaren gain eratua, [El euskera literario conforme al laburdino clásico] donde pergeña las bases de lo que será el euskera unificado, tema que ya había sido tratado en el Primer Congreso de Estudios Vascos de 1918, organizado por Eusko Ikaskuntza.

Villasante, que hizo diversas ediciones de Axular, y de otros clásicos, a la pregunta de por qué los clásicos respondió que porque son éstos los mejores testigos de la lengua, los que debemos conocer, analizar y usar, a fin de que las raíces de la misma lengua ahonden más en nuestro ser. Era en una encuesta del año 77 uno de los diez autores preferidos, y su diccionario de Axular una de las obras más leídas. Realmente hay que confesar que los que intentábamos escribir en la lengua estandar utilizamos bastante dicho léxico. 
El modelo leizarraguiano, pues, no tuvo mucho éxito. Krutwig, - $-\mathrm{y}$ algunos de sus seguidores posteriormente-, intentó promoverlo. En mayo del año 52 en su discurso de respuesta a Villasante, se alineó con la proposición de éste, pero los componentes políticos del mismo disgustaron a las autoridades vizcainas de tal manera que tuvo que exiliarse. En el exilio de París tomó contacto con escritores como Andima Ibiñagabeitia y Jon Mirande y juntos crean en 1953 el Pen Euskal Klub. Y escribió uno de los libros que según Mitxelena, ha sido uno de los que mayor daño ha hecho al País Vasco, Vasconia (1963), mezcla delirante de nacionalismo radical y de manual de guerrilla tercermundista. Aunque habría que matizar y puntualizar que probablemente en las cárceles franquistas de los años 60 se leyó bastante más la novela histórica de Navarro Villoslada, titulada Amaya o los vascos en el siglo VIII, e incluso, lo que es curioso sigue reeditándose actualmente con bastante éxito, lo que considera como patológico JoséCarlos Mainer (2000). A la vuelta del exilio ya no le convencían las fórmulas del euskara batua [euskera unificado], y el idiolecto que propuso, el krutwigiano, mezcla de euskara clásico y de griego a expuertas, y de escribir todos los términos cultos según los modelos greco-latinos, estaba tan alejado del habla del pueblo que sus novelas se le indigestaban hasta al mayor militante de la lengua.

Las propuestas de Michelena y Lafitte fueron bastante más sensatas y aunque personalmente no tenían ninguna dificultad para adoptar aquel sistema, -los dos eran profesores de una vasta cultura-, vieron que la mayoría de vascoparlantes tendría dificultades invencibles, por lo que prefirieron el modelo español al francés.

Pedro de Aguerre «Axular» con su obra Gero (1643), —-traducida al catalán con un estudio de Jean Haritschelhar-, fue considerado como el modelo a seguir. El maestro para los escritores del XVII, pero también para los del XVIII como Etxeberri de Sara, y del XIX como Chaho.

Antoine d'Abbadie al hablar de la obra y del prólogo, dice, que contiene frecuentes citas de clásicos sagrados y profanos, lo que produce una variedad de erudición y de pensamiento que se encuentra raramente en los libros de ascética. El estilo es simple y bello, y la dedicatoria a Bertrand d'Etchauz, arzobispo de Tours, llena de candor, elocuencia y fe. Ya lo indicaba el censor Pedro d'Urthubie, al decir que unía a su gran facundia la erudición, «atque uberem authoris facundiam magna cum erudictione».

Axular es un autor que tiene bastante relación con Fray Luis de Granada, por ejemplo y con otros autores renacentistas y barrocos, y el profesor Patxi Salaberri (1998) ha publicado una colección de exempla profanos que aparecen dispersos en el Gero. Los exemplos y fuentes clásicas que ornaban los sermones y libros de la época también han sido señalados por Jean Baptiste Orpustan (1996) al hablar de Tartas, o por Inaxio Mujika (1996) al reeditar la obra de Agirre de Asteasu.

En un sermón inédito de la época que edité en el homenaje a Axular (1999) encuentro las mismas características, el mismo modo y el mismo color en esa varietas ingeniorum, variedad de recursos (descripciones, recursos patéti- 
cos, etc.) y que los jesuitas denominaban la corriente asianista, que dejaba al predicador ante la variedad de modelos, crear el suyo propio.

Claro que ya desde el siglo XV fueron abundantes las ediciones de las Artes praedicandi como la editada en la imprenta de Martinus Herbipolensis de Leipzig, o la de Etienne Hoert editada en Estrasburgo en 1513.

Como hemos señalado el doctor Joannes Etcheberri de Sara en su obra de 1712 Laburdiri Escuararen hatsapenac [Principios del euskara a Lapurdi] editada en 1907 por Julio de Urquijo tiene un capítulo que se titula, P. AXULAR, Saraco errotora escuarazco autoretaric hautuena eta famatuena [Pedro de Axular el vicario de Sara, el más selecto y famoso entre los autores vascos].

Siguiendo esta línea Agustín Chaho decía, aun siendo él suletino: Le basque de Sare, de Saint-Jean-de-Luz et de la côte, voilà le dialecte qu'il faut étudier de préferance, qu'on doit être jaloux de conserver et de reproduire sans cesse. El vasco de Sara, San Juan de Luz y de la costa, he aquí el dialecto que hay que estudiar preferentemente, que hay que conservar celosamente, y reproducir sin cesar. Es decir, el modelo labortano clásico.

En el siglo XVIII también el jesuíta Agustín de Cardaberaz escribió una obrita titulada Eusqueraren berri onac eta ondo escribitceco, ondo iracurteco ta ondo itzeguiteco erreglac... [Noticias del eusquera y Reglas para hablar, leer y escribir bien...] (1761). En esta obra también la referencia de obras clásicas es constante, y no faltan las de Quintiliano, Jerónimo, Agustín de Hipona, etc. Cardaveraz cita las semejanzas del euskera con el latín, compara los dialectos vascos con los griegos, considera que a los jóvenes vascos les es más fácil el latín que el castellano, etc. Pero la finalidad del librito es la de presentar el euskera como una lengua de cultura, y propone la naturalidad como fórmula, seguir a los clásicos, a quienes les viene de sí su retórica natural, considera que ha de ser también la ars rethorica vasca, ya que la validez de la retórica latina era universal. Este librito sirvió también para que el mundo greco-latino fuera algo más conocido entre los vascos.

Luis Luciano Bonaparte, sobrino del emperador Napoleón I, gran romanista y dialectólogo que publicó en Londres en 1863 la famosa Carte des Sept provinces basques. La délimitation actuelle de l'escuara et sa division en dialectes, sous-dialectes et variétés, publicó también diversas obras como la Biblia completa en euskera (1859) traducida por Jean Duvoisin, y dos años antes unos Diálogos vascongados en guipuzcoano, vizcaino, laburdino, suletino, francés y español (1857).

Unos años más tarde en el 1884 editó Arturo Campión una voluminosa gramática de los cuatro dialectos literarios, dando en el apéndice del segundo tomo textos con notas de:

A. Dialecto guipuzcoano: F. I. Lardizabal, Testamentu zarreco eta berrico kondaira (1855).

B. Dialecto labortano: P. Axular, Gero (1643). 
C. Dialecto suletino: J.B. Archu, Lafontainaren alegia bereziak (1848); E. Inchauspe, Jesu-Kristen Imitacionia (1883).

D. Dialecto vizcaino: J.A. Mogel, Peru Abarca (1880); F. Arrese-Beitia, Olerkiak (s. XIX).

Claro que en esta selección de autores, obras, géneros y dialectos faltan bastantes puntos dignos de reseñarse. En primer lugar faltan los dialectos navarros, tan bien estudiados por Bonaparte. Autores altonavarros y bajo navarros de primera calidad como: Etxepare, Mendiburu, Lopez, Leizarraga de Elkano,...

Mendiburu, Cardaveraz, Larramendi y Palacios fueron de los predicadores más famosos del siglo XVIII en el País Vasco, y fuera de él. La base sobre la que reposa el sermón es evidentemente la Biblia y más exactamente el Nuevo Testamento, siendo otras fuentes complementarias los primitivos padres de la Iglesia y los autores clásicos como Plinio el Joven y Cicerón. El uso de citas latinas es constante, aunque siempre viene a renglón seguido la traducción vasca generalmente no literal, sino libre. Las referencias de Mendiburu a la antigüedad clásica no se hallan en función de un mayor conocimiento de ésta sino de ser un elemento válido para la educación moral. Y la riqueza de Mendiburu no se halla en la variación de la composición, que siempre es semejante sino en la riqueza léxica y sintáctica.

Hacia el final del siglo XIX y comienzos del XX con el surgir del periodismo vasco se desarrollan fundamentalmente tres modelos.

El modelo navarro-laburdino propuesto por Jean Hiriart-Urruty (Hazparne, 1859-1915) que dirigió el semanario Escualduna de 1905 a 1915, periódico que duró hasta el año 1944. Autor de mayor interés como estilista que como ideólogo, ya que era un católico conservador y beligerante. Línea que siguió en cuanto al estilo el médico Jean Etchepare, no así respecto a los temas, ya que en su obra de 1910 titulada Buruchkak defendía de modo bastante liberal las relaciones amorosas.

El modelo vizcaino promovido por R.M. de Azkue en su Euskaltzale (18971899), totalmente en euskera, por lo que fue prohibida, que contiene una cantidad de textos literarios muy interesante. A la que siguió el diario político bilingüe Euzkadi (1913-1937) con 7.637 números.

El modelo guipuzcoano promovido desde Euskal Erria (1880-1918) con 1.215 números, a la que siguió el semanario Argia (1921-1936).

Y no hay que olvidar el órgano de la Academia de la Lengua Vasca-Euskaltzaindia, Euskera, que tuvo una edición bastante irregular desde 1919 hasta 1936, pero en la que se promovió la unificación de la lengua y en la que Azkue propuso el modelo denominado gipuzkera osotua, los años 34 y 35. Modelo sobre el que escribió su novela Ardi Galdua [La oveja perdida] (1918), así como Pierre Lhande su Yolanda (1921), siendo como eran el uno labortano y el otro vizcaino. Era evidentente que las razones sociolingüísticas pesaban mucho. ¿En que consistía este modelo? Simplemente la mayonesa que puso Lizardi en práctica, más en su prosa que en su poesía, fue un acercamiento del guipuzcoano al labortano clásico con unas pocas cucharadas de aceite vizcaino. 
Todo esto y con más detalle se puede leer en la obra del Villasante (1970), titulada, Hacia la lengua literaria común, y en la tesis de Koldo Zuazo (1988) sobre el proceso de unificación del euskera.

Pero hablemos de los modelos no sólo lingüísticos sino también literarios aunque sea de modo breve.

En cuanto a la poesía, podemos comparar la poesía de tradición oral con la poesía culta o elaborada, pero muchas veces las oposiciones y los límites entre lo que los alemanes llamaban la Naturpoesie y la Kunstpoesie no son muy precisos y la influencia de la primera en la segunda es evidente, como se puede comprobar en la canción medieval de Doña Emilia, reelaborada en el primer tercio del siglo XX por Lauaxeta.

Si nos referimos al cuento tradicional y al cuento literario, la influencia y persistencia tanto del modelo tradicional como de su temática en el cuento actual es también evidente, aunque habría que establecer muchas más variaciones. Así el cuento de las lamias, «las doines d'aigua» de Cataluña, que aparecen junto a fuentes y que se constata en la toponimia vasca ya desde el año 1025 en el cartulario de San Millán de la Cogolla en el texto que dice, «de illo fonte qui vocatur lamiturri» [de aquella fuente que llaman fuente de las lamias] tiene diversos modelos, como el recogido por Wentworth Webster en su Basque Legends (1877), traducido del inglés al castellano por Julio Velasco (Miraguano, 1989), y editado el original después de sus traducciones al inglés, al francés y al español, ciento veinte años después por Xipri Arbelbide (1993).

Uno de los cuentos de lamias ha sido reelaborado por Jean Elissalde «Zerbitzari» (1833-1961), prosista labortano extraordinario, autor además de una crónica sobre la primera guerra mundial en la que participó, en el estilo realista más rudo y crudo, y al margen del modelo costumbrista generalizado en la narrativa.

Del teatro popular del que son expresión las pastorales suletinas (Urquizu: 2008) podemos considerar que son arreglos y adaptaciones de misterios, novelas de caballerías y en general de la literatura de colportage francesa, literatura extendidísima por toda Europa. Y como modelo de teatro culto podemos considerar las propuestas teatrales de Gabriel Aresti y Bernardo Atxaga. Ambos autores siguen el modelo unificado, aunque el último es posterior al 68.

Del ensayo religioso y retórico ya hemos hablado al mencionar a Axular y a Cardaveraz, pero tenemos en el siglo XIX además del ensayo político, generalmente traducido, un ensayo de cosmogonía como es el titulado Azti Begia de 1834, del filósofo y periodista Agustín Chaho pero que se puede considerar como un modelo aislado, así como la obra mencionada Buruxkak (1910) del médico bajonavarro Jean Etchepare en la que unos de los artículos trataba del amor libre, pero que él mismo expurgó posteriormente a instancias de su mujer. 
Y no podemos olvidar, por supuesto el modelo que supone el profesor Mitxelena en su presentación de las bases de la Unificación en el texto de Aránzazu, ya que aunque Axular valía para el siglo XVII, las necesidades del hombre vasco del siglo XXI son harto diferentes, pero como podemos ver en el texto elegido y en las palabras expresas de Koldo, la cadena no se ha roto, ya que el eslabón y el viejo lazo entre los de antes y los de ahora sigue en pie, adecuándose a la nuevas circunstancias, vertiendo vino nuevo en el viejo odre.

\section{BIBLIOGRAFÍA}

Arbelbide, X. (1993 ed.) Wentworth Webster, Ipuinak, Euskal Klasikoak, Donostia, Kriseilu.

Bonaparte, L. L. (1857 ed.) Dialogues basques guipuscoans, biscaïens, labourdins, souletins par A.P. Iturriaga, J. A. de Uriarte, J. Duvoisin et E. Inchauspe, Londres, W.H.Billing.

Campión, A. (1884) Gramática de los cuatro dialectos literarios de la lengua euskara, Tolosa, E. López. [Reed. Bilbao, La Gran Enciclopedia vasca, 1977].

Chaho, J. A. (1854) Le Messager de Bayonne, 4-I-1854, n. ${ }^{\circ} 386$, p. 2.

Elissalde, J. «Zerbitzari» (1921) «Artho xuritzetan», Baiona, Gure Herria.

Fokkema, D. (1998) «La literatura comparada y el nuevo paradima», in Dolores Romero (ed.), Orientaciones en literatura comparada, Madrid, Arco, p. 153.

Fuster, F. (1969) Poetas, moriscos y curas, Madrid, Ciencia Nueva.

Krutwig, F. (1979) Vasconia, Bilbao, Biblioteca de autores vascos.

Lafitte, P. (1944) Grammaire basque (Navarro-labourdin littéraire), Bayonne, Ikas. [Reed. Donostia, Elkar, 1966].

Leizarraga, J. (1571) Jesus Christ gure Jaunaren Testamentu Berria, La Rochelle, Pierre Hautin.

Leys, S. (1998) L'Ange et le Cachalot, Paris, Seuil.

Lotman, Y. (1978) Estructura del texto artístico, Madrid, Istmo.

Mainer, J. C. (2000) La escritura desatada. El mundo de las novelas, Madrid, Tanto por saber.

Michelena, L. (1963) Lenguas y protolenguas. Filosofía y Letras, XVII, n. ${ }^{\circ} 2$, Salamanca, Univ. Salamanca.

Mujika, I. (1996 ed.) Agirre. Arpoi baten eran, Klasikoak, Irun, Alberdania. 
Olaziregi, M. J. (2001) «Canonical and non-canonical narrative in the basque context», Revista Internacional de Estudios Vascos, vol 46, Donostia, pp. 325-336.

Orpustan, J. B. (1996) «La Fable dans la littérature basque», Lapurdum I. Études Basques, Bayonne.

Salaberri, P. (1998) Axularren historiak, Iruña, Pamiela.

Tötösy, S. (1998) «Estudios postcoloniales: El “otro”, el sistema y una perspectiva personal o esto (también) es literatura comparada», in D. Romero (ed.), Orientaciones en literatura comparada, Madrid, Arco, pp. 109-207.

Urkiaga, E. «Lauaxeta» (1935) Arrats beran. Bilbao, Verdes.

Urkizu, P. (1992 ed.) Agosti Chaho, Azti-begia eta beste izkribu zenbait, Euskal Klasikoak, Donostia, Elkar.

Urkizu, P. (1995 ed.) Jean Elissalde «Zerbitzari», LVIIa gerlan, Irun, Alberdania.

Urkizu, P. (1999) «Axularren garaiko sermoi argitaragabe bat: erunt signa in sole et luna et stellis. L.21., Goure Ginco omnipotantac... aurkezpena, oharrak eta transkripzioa» Lapurdum IV, Bayonne, pp. 187-212.

Urkizu, P. (2000 ed.) Andima Ibiñagabeitia, Erbestetik barne-minez. 1935-1967. Zarautz, Susa.

Urquizu, P. (2007 ed.) Teatro popular vasco. Manuscritos inéditos del siglo XVIII. Estudio y edición. Madrid, UNED.

Villasante, L. (1952) «Literatur-euskera, laphurtarr klassikoaren gain eratua», Boletín de la Real Sociedad Vascongada de los Amigos del País, Donostia, pp. 87-119; 259-298.

Villasante, L. (1970) Hacia la Lengua Literaria común, Oñate, Aranzazu.

Villasante, L. (1973) Axularren hiztegia, euskara-español-français, Oinati, Jakin.

Zuazo, K. (1988) La unificación de la lengua vasca, Iker, Bilbo, Euskaltzaindia. 